\title{
Near-band-edge recombinations in multiwalled boron nitride nanotubes: Cathodoluminescence and photoluminescence spectroscopy measurements
}

\author{
P. Jaffrennou, ${ }^{1,2,3}$ J. Barjon, ${ }^{4}$ T. Schmid, ${ }^{2}$ L. Museur, ${ }^{5}$ A. Kanaev, ${ }^{6}$ J.-S. Lauret, ${ }^{3}$ C. Y. Zhi, ${ }^{7}$ C. Tang, ${ }^{7}$ Y. Bando, ${ }^{8}$ \\ D. Golberg, ${ }^{7,8}$ B. Attal-Tretout, ${ }^{2}$ F. Ducastelle, ${ }^{1}$ and A. Loiseau ${ }^{1}$ \\ ${ }^{1}$ Laboratoire d'Etudes des Microstructures, ONERA-CNRS, BP 72, 92322 Châtillon Cedex, France \\ ${ }^{2}$ Département de Mesure Physique, ONERA, Chemin de la Hunière, 91761 Palaiseau Cedex, France \\ ${ }^{3}$ Laboratoire de Photonique Quantique et Moléculaire, Institut d'Alembert, Ecole Normale Supérieure de Cachan, \\ 61 avenue du Président Wilson, 94235 Cachan Cedex, France \\ ${ }^{4}$ Groupe d'Etude de la Matière Condensée, Université de Versailles St Quentin, CNRS Bellevue, 1 place Aristide Briand, \\ 92195 Meudon Cedex, France \\ ${ }^{5}$ Laboratoire de Physique des Lasers, CNRS-UMR 7538, Université Paris XIII, Institut Galilée, 99 Avenue Jean-Baptiste Clément, \\ 93430 Villetaneuse, France \\ ${ }^{6}$ Laboratoire d'Ingénierie des Matériaux et des Hautes Pressions, CNRS, Institut Galilée, Université Paris XIII, \\ 93430 Villetaneuse, France \\ ${ }^{7}$ Nanoscale Materials Center, National Institute for Materials Science, Namiki 1-1, Tsukuba, Ibaraki 3050044, Japan \\ ${ }^{8}$ World Premier International Center for Materials Nanoarchitectonics (MANA), National Institute for Materials Science, Namiki 1-1, \\ Tsukuba, Ibaraki 3050044, Japan
}

(Received 4 April 2008; published 16 June 2008)

\begin{abstract}
Individual multiwall boron nitride nanotubes with diameters from $30 \mathrm{~nm}$ to $110 \mathrm{~nm}$ are shown to be efficient UV emitters by cathodoluminescence. Their luminescence does not evolve much in this diameter range, with dominant UV recombinations at about $230 \mathrm{~nm}$. As a result, single nanotube properties can be obtained from experiments performed on ensembles of nanotubes. Such ensembles are studied by photoluminescence as a function of temperature $(5 \mathrm{~K}-300 \mathrm{~K})$ and by photoluminescence excitation experiments at $9 \mathrm{~K}$. The results are discussed and compared with the related bulk material, hexagonal boron nitride. The strong luminescence recorded around $230 \mathrm{~nm}$ is attributed to excitonic effects, more precisely to excitons bound to the structural defects: dislocations, facets, which are observed along the walls.
\end{abstract}

DOI: $10.1103 /$ PhysRevB.77.235422

PACS number(s): 78.67.Ch, 71.35.-y, 71.55.Eq, 78.55.Cr

\section{INTRODUCTION}

Since the early 1990s, much interest has been devoted to nanotubes and especially to carbon nanotubes. ${ }^{1}$ In 1994 , theoretical studies have predicted that boron nitride (BN) nanotubes would be stable and that their electronic properties would be very different from those of carbon nanotubes. ${ }^{2,3}$ According to these calculations, BN nanotubes are expected to be wide band-gap semiconductors and their band gap should not depend significantly on their diameter and chirality. A few years after the first calculations, multiwalled ${ }^{4,5}$ and then, single-walled ${ }^{6,7} \mathrm{BN}$ nanotubes were synthesized. However, up to now, only few experiments are reported on BN nanotubes and most of them are performed on as-grown macroscopic samples.

Recent theoretical calculations on single-walled BN nanotubes ${ }^{8,9}$ have demonstrated that, as in the case of the bulk material, hexagonal boron nitride $(\mathrm{hBN}),{ }^{10} \mathrm{BN}$ nanotubes should show huge excitonic effects with a large binding energy typical of Frenkel-like excitons. Thus, even if the band gap of BN nanotubes should not vary too much with diameter, it is necessary to perform measurements on individual nanotubes in order to obtain a good understanding of their intrinsic properties. Some experiments on such nanotubes have already been reported concerning their structural, ${ }^{11-16}$ mechanical, ${ }^{17}$ and electronic properties. ${ }^{18-21}$ However, up to now, very few studies have dealt with the optical properties of individual BN nanotubes. Electron energy-loss spectroscopy in the low loss range has been performed on individual single-walled and multiwalled $\mathrm{BN}$ nanotubes ${ }^{12}$ but the optical transitions are indirectly probed. Cathodoluminescence, ${ }^{22}$ photoluminescence, ${ }^{23}$ and opticalabsorption measurements ${ }^{24}$ on $\mathrm{BN}$ nanotube macroscopic samples have also been reported. In the luminescence studies, ${ }^{22,23}$ a broad luminescence band around $350 \mathrm{~nm}$ was observed and was erroneously attributed to interband transitions.

Concerning optical absorption measurements, two absorption bands were reported around 280 and $225 \mathrm{~nm}$ and were tentatively assigned to excitonic levels and Van Hove singularities, respectively. ${ }^{24}$ By comparing optical absorption and luminescence spectra on multiwalled BN nanotubes, ${ }^{25}$ another interpretation of these results has been proposed: the $280 \mathrm{~nm}$ band is attributed to the absorption band of impurity levels whereas the $225 \mathrm{~nm}$ band is attributed to the near-edge excitonic absorption. Recently, we have also performed cathodoluminescence spectroscopy and imaging on individual bamboolike $\mathrm{BN}$ nanotube ${ }^{26}$ and we observed that, as expected, these nanotubes were strong UV luminescent materials. A very recent cathodoluminescence study on ensembles of BN nanotubes with different boron isotopes has also been reported and confirms that $\mathrm{BN}$ nanotubes are UV emitters. ${ }^{27}$

In this paper, we present a study of the optical properties of multiwalled $\mathrm{BN}$ nanotubes previously characterized by transmission electron microscopy (TEM). The experimental 
details are presented in Sec. II. Then, individual BN nanotubes of different diameters are analyzed by cathodoluminescence (CL) spectroscopy and imaging (Sec. III). Photoluminescence (PL) at various temperatures and photoluminescence excitation (PLE) spectroscopies are also performed on ensembles of tubes (Sec. IV). These observations will allow us to discuss the origin of the luminescence of multiwalled nanotubes (Sec. V).

\section{EXPERIMENTS}

The BN nanotube sample is composed of stoichiometric multiwalled nanotubes synthesized by chemical vapor deposition using boron oxide as reactant ${ }^{28,29}$ and purified by a polymer wrapping process. ${ }^{30}$ The resulting powder is white and is composed of a high concentration of multiwalled BN nanotubes with diameters ranging from about 20 to $150 \mathrm{~nm}$. $\mathrm{hBN}$ powders are high-quality commercial powders (Aldrich Corp.) already described elsewhere. ${ }^{31,32}$ For CL analysis, the sample is dispersed in ethanol, deposited on carbon coated TEM copper grids, and observed with a Philips CM20 TEM at an accelerating voltage of $200 \mathrm{keV}$. CL measurements are performed in a JEOL840 scanning electron microscope (SEM) with a $20 \mathrm{keV}$ electron beam and using the experimental setup described in a previous paper. ${ }^{32}$ The spatial resolution of the CL images is limited by the beam size of about $0.3 \mu \mathrm{m}$. For PL and PLE measurements, the powder is deposited on UV transparent substrates. An ArF excimer laser emitting at $193 \mathrm{~nm}$ (repetition rate $25 \mathrm{~Hz}$, pulse duration 3.5 ns) is used for PL excitation. The sample is inserted in a variable-temperature cryostat (ST-100, Janis Research Co.). An achromatic triplet lens collects the light in a backscattering configuration and the emitted spectra are dispersed using a SpectraPro 2558i spectrometer equipped with three gratings. The signal is detected with a charge coupled device (CCD) camera. According to the grating used, the spectral resolution can reach $0.05 \mathrm{~nm}$. The PL and CL spectra are corrected from the instrumental response, measured with a calibrated deuterium lamp. PLE spectroscopy is carried out at the SUPERLUMI station at HASYLAB, DESY synchrotron-radiation source (Hamburg, Germany). The facility of the SUPERLUMI station used for experiments is described in details elsewhere. ${ }^{33}$ The spectral resolution of the setup is $1.3 \mathrm{~nm}$.

\section{SINGLE TUBES}

The CL spectra of four individual BN nanotubes of outer diameters equal to 30, 60, 75, and $110 \mathrm{~nm}$ are shown in Fig. 1. The TEM images of each BN nanotube reveal that they are composed of about 25, 50, 60, and $120 \mathrm{BN}$ layers, respectively. In Fig. 1, their light-emission properties are compared with the spectrum of an ensemble composed of a few hundreds of nanotubes with various diameters, as assessed by TEM and SEM. We observe that all spectra show two main luminescence signals whose maxima are centered at about 233 and $320 \mathrm{~nm}$. It is remarkable that the CL spectrum of the ensemble and those of the individual BN nanotubes can almost be superimposed. From the experimental point of view,

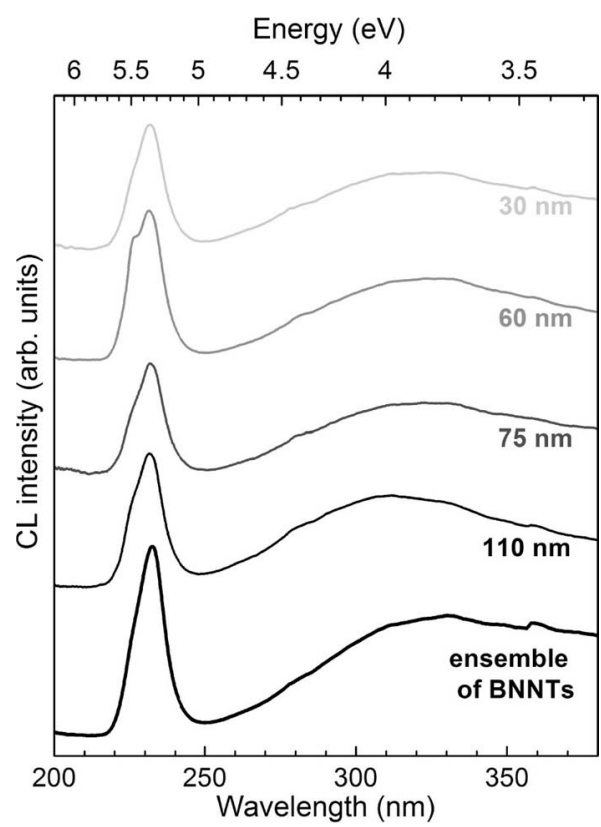

FIG. 1. Top: CL spectra of individual multiwalled BN nanotubes with outer diameters equal to $30,60,75$, and $110 \mathrm{~nm}$ (inner diameters: $23,45,55$, and $70 \mathrm{~nm}$ ); $T=100 \mathrm{~K}, I=2.8 \mathrm{nA}$. Bottom: CL spectrum of an ensemble of BN nanotubes; $T=100 \mathrm{~K}, I=1.8 \mathrm{nA}$.

this means that a macroscopic study on an ensemble of tubes is relevant for studying the properties of single tubes.

Examining the spectra in more detail, we see that the dominant luminescence signal at higher energy displays two peaks at $232.5 \mathrm{~nm}$ and at $225 \mathrm{~nm}$. This is particularly striking on the luminescence spectrum of the $60 \mathrm{~nm}$ diameter nanotube. We do not observe a significant broadening of the linewidth of the CL high energy luminescence band when comparing the ensemble of nanotubes and individual nanotubes $(\mathrm{FWHM}=0.3 \mathrm{eV}$ ), which confirms that this width is not dominated by the inhomogeneities among different nanotubes. If we compare these results with the excitonic luminescence observed in $\mathrm{hBN}^{32,34}$ and with the recent theoretical predictions for nanotubes, ${ }^{8,9}$ we can assume that this high energy band is not related to simple interband transitions, but rather to near band-gap excitonic recombinations.

The $320 \mathrm{~nm}$ broad band has already been observed in luminescence spectra of multiwalled BN nanotubes, ${ }^{22,25,26,35}$ $\mathrm{BN}$ nanorods, ${ }^{36,37}$ and $\mathrm{BN}$ whiskers ${ }^{38}$ and has been attributed either to impurity and defect centers or to radiative excitonic dark states..$^{9,24,35}$ If we compare this band with that observed in bamboolike $\mathrm{BN}$ nanotubes, ${ }^{26}$ we can identify it as an impurity-related luminescence band. We have also observed that, for individual $\mathrm{BN}$ nanotubes, the relative intensities of this emission band and of the UV peaks centered on $233 \mathrm{~nm}$ are very dependent on the crystalline quality of the nanotube, as characterized by TEM. This is also what has been observed in $\mathrm{hBN}$ when comparing the impurity band with the excitonic one. ${ }^{31,34,39}$

In order to explore more deeply the nature of both luminescence bands, monochromatic CL images of isolated $\mathrm{BN}$ nanotubes have been recorded (Fig. 2). Previous observations of polychromatic CL images of individual $\mathrm{BN}$ 


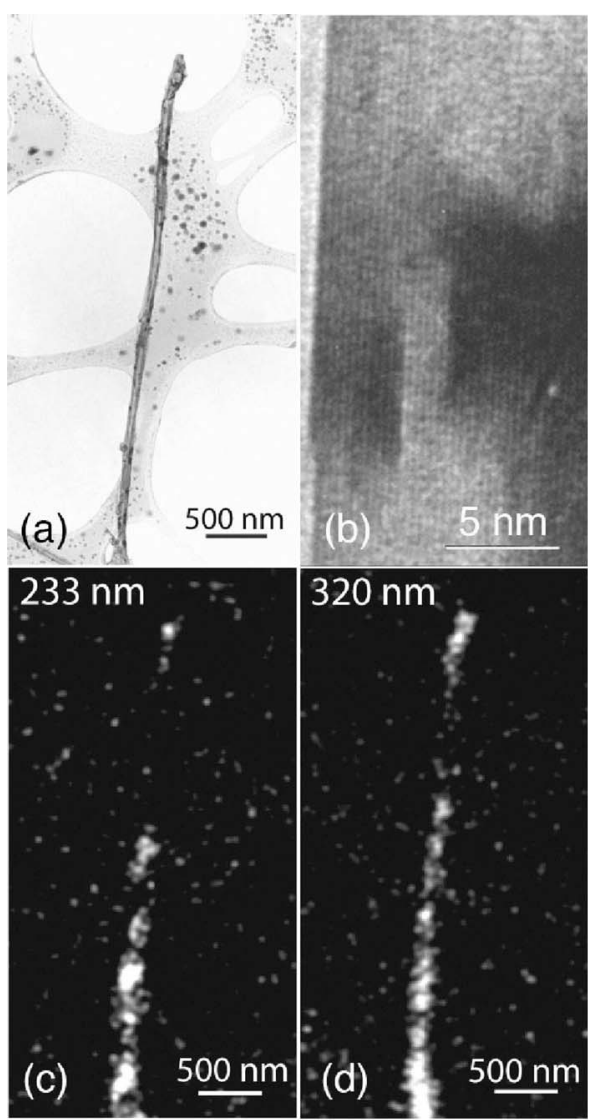

FIG. 2. (a) TEM image, (b) High-resolution TEM (HRTEM) of the nanotube, (c) monochromatic CL images at $233 \mathrm{~nm}$ and (d) at $320 \mathrm{~nm}$ of a $75 \mathrm{~nm}$ diameter BN nanotube. CL images are taken at $T=100 \mathrm{~K}$ and $I=700 \mathrm{pA}$.

nanotubes ${ }^{25,26}$ have shown that the total luminescence is located all along the nanotube and that it is quite homogeneously distributed over the tube. Figures 2(a)-2(d) display TEM images of the $75 \mathrm{~nm}$ diameter BN nanotube and monochromatic images of the same nanotube centered at 233 and $320 \mathrm{~nm}$. We observe that the monochromatic CL images do not exactly show the same light spatial distribution: The luminescence at $320 \mathrm{~nm}$ is emitted quite homogeneously along the tube whereas the luminescence at $233 \mathrm{~nm}$ seems to be brighter on specific areas of the nanotube. TEM images at high magnification indicate that the luminescence at $233 \mathrm{~nm}$ is quenched where the tube is in contact with the amorphous carbon membrane.

Figure 3 shows the comparison between a monochromatic CL image taken at $233 \mathrm{~nm}$ and a TEM image of another nanotube whose outer diameter is equal to $60 \mathrm{~nm}$. These images confirm the last observation since, as indicated by the rectangles, the light emitted at $233 \mathrm{~nm}$ is more diffuse and much less intense on areas where the nanotube is laid on the amorphous carbon film; thus, light is mainly detected where the nanotube is suspended over holes of the carbon membrane. This weakening of the luminescence observed when the tube is in contact with the membrane can be attributed to a charge transfer between the semiconducting tube and the conductive substrate. This phenomenon has already been observed when semiconducting carbon nanotubes are in contact

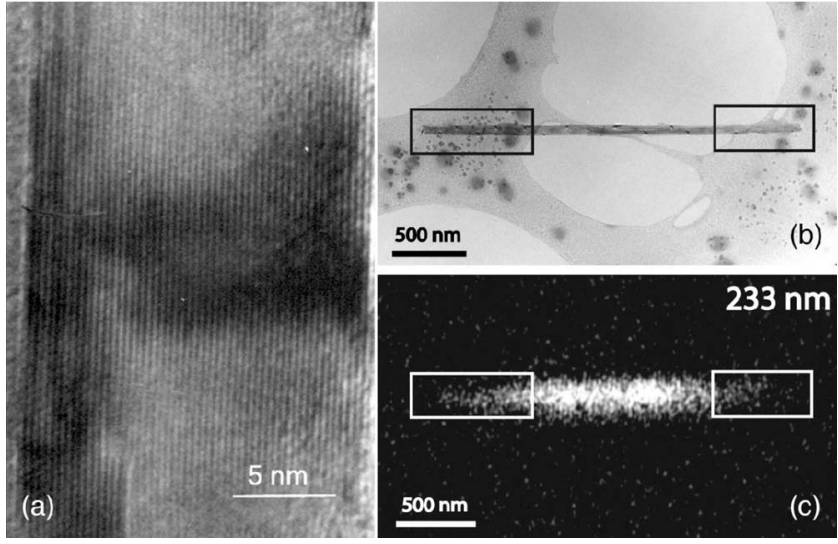

FIG. 3. (a) HRTEM, (b) TEM image, and (c) monochromatic CL image at $233 \mathrm{~nm}$ of a $60 \mathrm{~nm}$ diameter $\mathrm{BN}$ nanotube. The empty rectangles indicate areas where the nanotube is laid on the amorphous carbon membrane. CL images are taken at $T=100 \mathrm{~K}, I$ $=700 \mathrm{pA}$.

with conductive materials such as metallic carbon nanotubes, organic molecules, or metallic substrates. ${ }^{40-43}$

\section{ENSEMBLES OF TUBES}

A concentrated ensemble of the same BN nanotubes have been analyzed at a macroscopic scale in PL spectroscopy excited at $193 \mathrm{~nm}(6.42 \mathrm{eV})$ and compared to an hBN powder at $5 \mathrm{~K}$. Figure 4 shows the superposition of PL spectra taken at $5 \mathrm{~K}$ for a deposit of $\mathrm{BN}$ nanotubes and of $\mathrm{hBN}$ powders. The PL spectrum of $\mathrm{BN}$ nanotubes is similar to those recorded in CL spectroscopy (Fig. 1) and is comparable to the PL spectrum of hBN. The PL spectrum of the macroscopic BN nanotube sample is composed of two luminescence bands: The UV band, that we attribute to excitonic recombinations, has a maximum PL intensity at $232 \mathrm{~nm}$ and the deep blue luminescence band, that we assign to impurity centers, is observed at about $310 \mathrm{~nm}$. Concerning $\mathrm{hBN}$, the impurity luminescence band is observed around $300 \mathrm{~nm}$ and

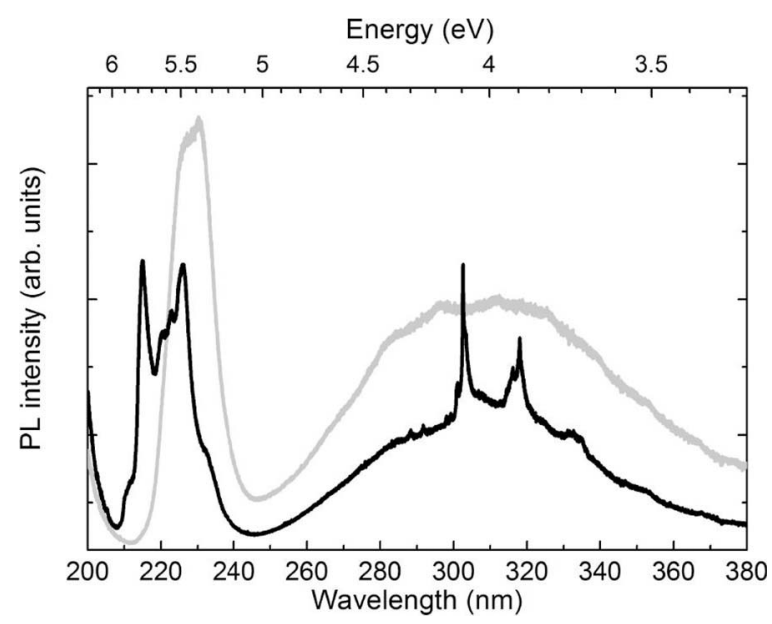

FIG. 4. PL spectra for a deposit of BN nanotubes (gray) and of hBN crystallites (black). $T=5 \mathrm{~K}$, excitation at $193 \mathrm{~nm}$. 


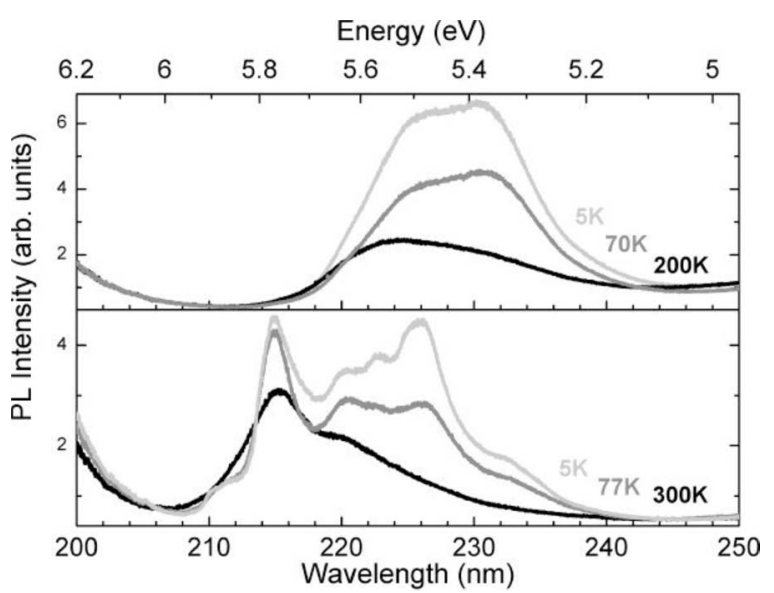

FIG. 5. PL spectra centered on the excitonic band of a deposit of $\mathrm{BN}$ nanotubes (top) and of $\mathrm{hBN}$ as a function of temperature (5-300 K) (down). Excitation at $193 \mathrm{~nm}$.

it is attributed to carbon or oxygen impurities. ${ }^{39}$ It shows strong phonon replica indicated on the spectrum at 304, 318, and $334 \mathrm{~nm},{ }^{31,35,44}$ whereas the BN nanotube impurity band is only composed of a wide band $(\mathrm{FWHM}=1.3 \mathrm{eV})$ at about the same wavelength. The absence of noticeable phonon replica in the impurity luminescence band of BN nanotubes has already been mentioned in another tubular $\mathrm{BN}$ material (bamboolike BN nanotubes). ${ }^{26}$ In this respect, the results obtained in a recent isotope study are fairly surprising: Strong phonon replica are observed in ${ }^{10} \mathrm{BN}$ nanotubes but not in natural $\mathrm{BN}$ nanotubes. ${ }^{27}$ The authors argue that narrower structures are expected for pure isotopes but on the other hand they do not observe phonon replica in their spectra corresponding to ${ }^{11} \mathrm{BN}$ nanotubes.

In order to further investigate the excitonic recombinations in both materials, PL spectra of BN nanotubes and hBN have been recorded at various temperatures, between 5 and $300 \mathrm{~K}$ (Fig. 5). Since the optical alignment is unchanged during macroscopic PL measurements, the PL intensities of the bands at all temperatures are directly comparable. As observed in Fig. 5, the PL intensities of the excitonic bands of both materials increase when lowering the temperature but the PL lines do not seem to shift.

For hBN, the excitonic spectrum is composed of at least three lines observed at 215, 220, and $227 \mathrm{~nm}$ with relative intensities depending on temperature (Fig. 5). It is now well admitted that the line at $215 \mathrm{~nm}$, which is the most intense at $300 \mathrm{~K}$ corresponds to free excitons..$^{32,34,45}$ As the temperature decreases, the intensities of the lines observed at 220 and 227 $\mathrm{nm}$ increase dramatically. These lines were previously assigned to excitons bound to structural defects, namely, grain boundaries and dislocations. ${ }^{32,45,46}$ The observed increase of the 220 and $227 \mathrm{~nm}$ lines at low temperature is indeed consistent with a mechanism of exciton trapping on defects, which is more efficient at low temperature. The hBN luminescence line at $234 \mathrm{~nm}$ is attributed to quasi-donor-acceptor pairs $(q$-DAP $) .{ }^{47}$

For BN nanotubes, the spectral behavior of the excitonic band as a function of temperature is quite different from that of hBN. The nanotube band seems to be composed of at least

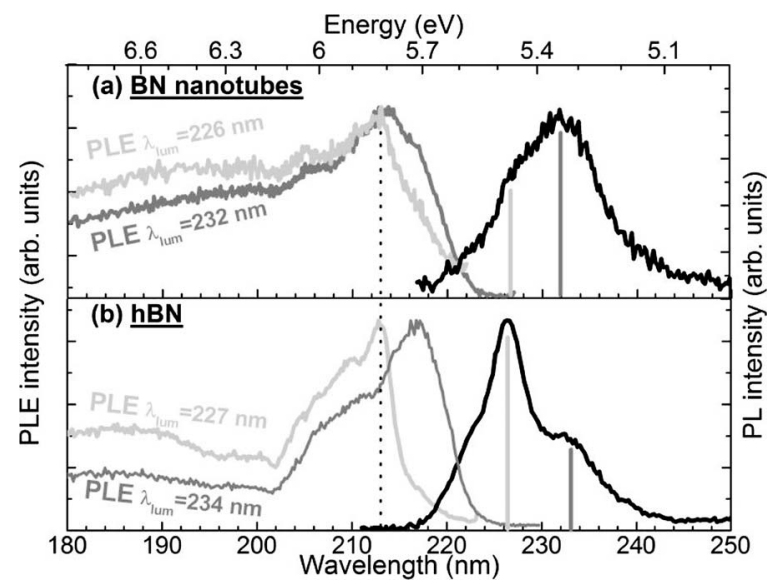

FIG. 6. PL and PLE spectra of (a) a deposit of BN nanotubes and (b) pressed hBN powders. The PL spectra (dark lines; logarithmic scale) are excited at $213 \mathrm{~nm}$. The normalized PLE spectra correspond to emissions at 226 and $232 \mathrm{~nm}$ in (a) and to $227 \mathrm{~nm}$ in (b) (gray lines); $T=9 \mathrm{~K}$.

two lines centered at about 224 and $232 \mathrm{~nm}$. This structure was also observed in CL spectra of individual BN nanotubes (See Fig. 1). While lowering the temperature, the PL UV band of BN nanotubes follows the same trend as the hBN excitonic band but the relative intensities of the lines do not change very much with temperature. As observed in Fig. 5, the maximum PL intensity is observed at about $224 \mathrm{~nm}$ at $200 \mathrm{~K}$ and at $232 \mathrm{~nm}$ at $5 \mathrm{~K}$. Unlike the case of hBN, we do not observe any noticeable and narrow line at high energy which could be assigned to free excitonic recombinations. The PL line of BN nanotubes at $232 \mathrm{~nm}$ is observed at a position quite close to that of the $q$-DAP line of hBN, at 234 $\mathrm{nm} \cdot{ }^{47}$ However, the strong increase of the PL intensity of this $232 \mathrm{~nm}$ line at low temperature seems to correspond to excitonic transitions more than to DAP transitions. This is confirmed by the analysis of PLE spectra, as discussed below. In addition, the nanotube excitonic band seems to be broader $(\mathrm{FWHM}=0.3 \mathrm{eV})$ than the one of $\mathrm{hBN}$, which is made up of well-defined lines at 215, 220, and $227 \mathrm{~nm}$ (Fig. 5).

In order to investigate this $\mathrm{BN}$ nanotube excitonic emission further, PLE spectroscopy combined with PL spectroscopy excited at $213 \mathrm{~nm}$ has been performed at $9 \mathrm{~K}$ on the same BN nanotube macroscopic sample. Figure 6(a) displays the PL spectrum centered on the excitonic band and the PLE spectra taken at 232 and $226 \mathrm{~nm}$, which correspond to the emission wavelengths observed on the PL excitonic band. The PLE spectra are similar and they both display a highest intensity peak at $213 \mathrm{~nm}$. This similarity indicates that the 226 and $232 \mathrm{~nm}$ luminescence lines may have the same origin. In order to analyze the PLE spectra, it is interesting to compare the PLE spectra of BN nanotubes with the PLE spectrum of deformed hBN (pressed powders) taken at 227 $\mathrm{nm}$ (bound exciton line) and at $234 \mathrm{~nm}(q$-DAP) obtained by Museur and Kanaev. ${ }^{47}$ As shown in Fig. 6(b), the maximum intensity of the PLE spectrum of hBN taken at $227 \mathrm{~nm}$ is also observed at $213 \mathrm{~nm}$ and it is then attributed to the free excitonic level which is observed at $215 \mathrm{~nm}$ in luminescence (Fig. 5). Notice here that deformed hBN do not emit at this wavelength ${ }^{45}$ (see also Fig. 6(b) and the discussion in Sec. 
$\mathrm{V})$. For the hBN spectrum taken at $234 \mathrm{~nm}$, the maximum is clearly at a larger wavelength. The fact that the maximum of the PLE spectrum of nanotubes taken at $232 \mathrm{~nm}$ is also at $213 \mathrm{~nm}$ shows that DAP transitions cannot be totally responsible for this PL $232 \mathrm{~nm}$ emission.

The comparison between $\mathrm{BN}$ nanotubes and hBN PLE spectra clearly indicates that they both display quite similar excitonic structures. Up to now, we do not have enough experimental data to assign the $\mathrm{BN}$ nanotubes 226 and $232 \mathrm{~nm}$ PL lines either to phonon replica, to substructures of an excitonic transition, or to different excitons bound to different types of traps.

\section{DISCUSSION}

Explaining these experimental results is not straightforward since there are no theoretical studies for multiwalled $\mathrm{BN}$ nanotubes dealing with their electronic structure or excitonic effects. However, according to theoretical calculations on $\mathrm{hBN}^{10}$ and single-walled $\mathrm{BN}$ nanotubes, ${ }^{8,9}$ these materials have quite similar optical properties and are expected to display huge excitonic effects, with large oscillator strength on excitonic levels. Then, comparisons with the optical properties of hBN support the explanations we can propose here for the experimental results on multiwalled $\mathrm{BN}$ nanotubes.

The first experimental result is that there is no difference between the luminescence spectra of individual BN nanotubes with diameters ranging from 30 to $110 \mathrm{~nm}$ (Fig. 1). This observation indicates that excitonic effects in multiwalled BN nanotubes do not vary as a function of their diameter and of their number of layers. There is no quantum confinement effect, which is consistent with the theoretical predictions that the excitons in $\mathrm{hBN}$ and $\mathrm{BN}$ nanotubes should be of Frenkel (localized) type. Our nanotubes are indeed composed of at least 20 BN layers (68 A thick). Since the multiwalled $\mathrm{BN}$ nanotubes are expected to behave as hBN in terms of excitonic effects, their Frenkel-type excitons are strongly confined in the layers, with spatial extension of a few atoms within these layers. ${ }^{10}$ This spatial extension is far below the size of the individual $\mathrm{BN}$ nanotubes studied here and this explains why we do not observe differences in the excitonic emission of different individual nanotubes.

The second observation is that the excitonic emission of $\mathrm{BN}$ nanotubes is red shifted by about $190 \mathrm{meV}$ at $300 \mathrm{~K}$ as compared to the free exciton line of hBN (Fig. 5). Such a result can be explained by a variation of the gap. However, thanks to PLE experiments, we see that the optical gap value is similar for $\mathrm{hBN}$ and $\mathrm{BN}$ nanotubes since the maximum of the PLE spectrum is observed at the same position. Then, we can assume that this red shift is due to differences in their excitonic recombinations, either because of polarization effects induced by the change in symmetry and geometry from $\mathrm{hBN}$ to $\mathrm{BN}$ nanotubes, or because of the very nature of the excitons responsible for this emission.

As far as the first assumption is concerned, it has been demonstrated theoretically and experimentally that BN materials are submitted to large internal electric fields, as shown by the observation of strong Stark effects in these systems. ${ }^{19-21,48}$ Furthermore, hBN and BN nanotubes are ex- pected to display piezoelectricity, as observed in most hexagonal III-N semiconductors, ${ }^{49}$ especially because of their hexagonal structure, i.e., of their polarity and anisotropy. As reported in other III-N materials, strain can induce stronger piezoelectric effects because of the break of symmetry, and this effect is often characterized by a red shift of the emission lines. ${ }^{50,51}$ As mentioned above, the nanotubes studied here have large diameters and several layers so that their crystalline structure can be assimilated to thin pieces of hBN with curvature. This curvature, which is also characteristic of bamboolike $\mathrm{BN}$ nanotubes and $\mathrm{BN}$ whiskers, induces additional strains which can be partially responsible for the red shifted excitonic emission of the BN nanotubes and also for the large linewidth of the luminescence bands. Further investigation of the $\mathrm{BN}$ nanotube piezoelectricity compared to that of $\mathrm{hBN}$ is required to understand more precisely the role of internal electric field on emission properties.

The second assumption is that the nature of the excitons responsible for this emission can explain the red shift. Indeed, such a red shift has also been reported in the CL spectra of hBN after deformation. Watanabe et al. ${ }^{45}$ observed that, after pressing a hBN single crystal between two fingers, the CL spectrum of the deformed hBN was dominated by a luminescence signal at $227 \mathrm{~nm}$ instead of the free excitonic luminescence at $215 \mathrm{~nm}$. This red shifted emission was attributed to changes in the nature of the excitonic recombinations in terms of excitons bound to the structural defects created by the mechanical deformation (see also Ref. 32). As far as BN nanotubes are concerned, high-resolution TEM images of the analyzed BN nanotube sample (see insets in Figs. 2 and 3) reveal that their walls are constituted of structural defects such as dislocations and stacking faults ${ }^{25,52}$ and a recent TEM and electron-diffraction analysis of the same sample indicate that the inner tubes are polygonized. ${ }^{53}$

These observations indicate that these $\mathrm{BN}$ nanotubes can be viewed as curved hBN with structural defects all along the tube. This description of multiwalled $\mathrm{BN}$ nanotubes can explain their luminescence properties and the observed red shift in the same way they are analyzed in the case of deformed hBN: The luminescence can be dominated by recombinations of excitons bound to structural defects. By comparing the spectral positions of the $\mathrm{BN}$ nanotube luminescence lines with the hBN bound exciton lines at 220 and $227 \mathrm{~nm}$, we see that the excitonic recombinations of $\mathrm{BN}$ nanotubes do not emit exactly at the same wavelengths as the $\mathrm{hBN}$ ones. An explanation for this slight difference in the energy position of the excitonic lines could be the presence of local electric fields induced by defects in $\mathrm{BN}$ nanotubes or other effects induced by the tubular geometry of the nanotubes. Other experimental studies ${ }^{35}$ suggest a possible interpretation of these BN nanotubes luminescence lines in terms of selftrapped excitons (STEs). STEs are usually observed in ionic crystals, molecular crystals, or alkali halides ${ }^{54}$ and are localized excitons with a large binding energy. STEs are formed from the relaxation of free excitons into lower energy levels where the excitons are trapped because of local distortions of the lattice due to local stress, defects, or charges. Further investigations of the relaxation processes in $\mathrm{hBN}$ and in $\mathrm{BN}$ nanotubes, using time-resolved luminescence spectroscopy, are required to decide between different possible interpreta- 
tions of the excitonic recombinations in $\mathrm{BN}$ nanotubes.

The last experimental result which supports the interpretation of the excitonic luminescence of $\mathrm{BN}$ nanotubes as due to bound excitons is the PLE spectra (Fig. 6). As mentioned above, the hBN PLE spectrum taken on the $227 \mathrm{~nm}$ luminescence also shows a maximum intensity at $213 \mathrm{~nm}$ which, in $\mathrm{hBN}$, and up to a small Stokes shift, can be accurately correlated with the free excitonic luminescence line observed at $215 \mathrm{~nm}$. As BN nanotubes and hBN display similar PLE spectra, we conclude that they have similar electronic structures and optical gaps, and that the differences in their UV luminescences are more likely due to excitonic effects than to variations in the gap value. This similarity indicates that the luminescence lines of BN nanotubes at 226 and $232 \mathrm{~nm}$ have the same nature than this $227 \mathrm{~nm}$ line in $\mathrm{hBN}$, i.e., they are due to bound exciton recombinations. As expected in $\mathrm{hBN},{ }^{47}$ these bound excitons recombine after relaxation from the free exciton which is formed at $213 \mathrm{~nm}$.

\section{CONCLUSION}

We have presented an experimental study of the optical properties of multiwalled $\mathrm{BN}$ nanotubes compared with those of the related bulk material, hBN. A combination of luminescence spectroscopies, imaging, and photoluminescence excitation spectroscopy on pure and very concentrated multiwalled $\mathrm{BN}$ nanotube samples have been presented. We have found that these nanotubes are light emitters around $230 \mathrm{~nm}$. On the basis of the analysis of the hBN luminescence properties, we explain this UV luminescence in terms of strong excitonic effects.

All experimental results suggest that multiwalled $\mathrm{BN}$ nanotubes with diameters in the range $30-110 \mathrm{~nm}$ and with a large number of layers can be considered as pieces of curved $\mathrm{hBN}$ where the optical properties are dominated by excitons bound to structural defects. These results are very promising in view of possible applications of $\mathrm{BN}$ nanotubes in optoelectronic devices or as solid-state light sources. ${ }^{55}$ The experimental method developed in this work, based on the combination of several optical techniques is a first step toward the investigation of single-walled BN nanotubes. This study as well as time-resolved spectroscopy measurements are under progress.

\section{ACKNOWLEDGMENTS}

The authors are grateful to N. Dorval and to S. Maine for technical support, to G. Stryganyuk for assistance in conducting experiments at SUPERLUMI station, and to R. Arenal, B. Arnaud, J.-D. Ganière, A. Rubio, and L. Wirtz, for interesting and fruitful discussions. This work has been supported by the GDR on "Science and Applications of Nanotubes" (GDRE2756), by the ANR PNANO project "Nanotube Suspendu," and by the IHP-Contract No. HPRI-CT1999-00040 of the European Commission.
${ }^{1}$ S. Iijima, Nature (London) 354, 56 (1991).

${ }^{2}$ A. Rubio, J. L. Corkill, and M. L. Cohen, Phys. Rev. B 49, 5081 (1994).

${ }^{3}$ X. Blase, A. Rubio, S. G. Louie, and M. L. Cohen, Europhys. Lett. 28, 335 (1994).

${ }^{4}$ N. G. Chopra, R. J. Luyken, K. Cherrey, V. H. Crespi, M. L. Cohen, S. G. Louie, and A. Zettl, Science 269, 966 (1995).

${ }^{5}$ A. Loiseau, F. Willaime, N. Demoncy, G. Hug, and H. Pascard, Phys. Rev. Lett. 76, 4737 (1996).

${ }^{6}$ R. S. Lee, J. Gavillet, M. Lamy de la Chapelle, A. Loiseau, J. L. Cochon, D. Pigache, J. Thibault, and F. Willaime, Phys. Rev. B 64, 121405(R) (2001).

${ }^{7}$ R. Arenal, O. Stéphan, J.-L. Cochon, and A. Loiseau, J. Am. Chem. Soc. 129, 16183 (2007).

${ }^{8}$ L. Wirtz, A. Marini, and A. Rubio, Phys. Rev. Lett. 96, 126104 (2006).

${ }^{9}$ C.-H. Park, C. D. Spataru, and S. G. Louie, Phys. Rev. Lett. 96, 126105 (2006).

${ }^{10}$ B. Arnaud, S. Lebègue, P. Rabiller, and M. Alouani, Phys. Rev. Lett. 96, 026402 (2006).

${ }^{11}$ D. Golberg, Y. Bando, M. Eremets, K. Takemura, K. Kurashima, and H. Yusa, Appl. Phys. Lett. 69, 2045 (1996).

${ }^{12}$ R. Arenal, O. Stéphan, M. Kociak, D. Taverna, A. Loiseau, and C. Colliex, Phys. Rev. Lett. 95, 127601 (2005).

${ }^{13}$ R. Arenal, M. Kociak, A. Loiseau, and D.-J. Miller, Appl. Phys. Lett. 89, 073104 (2006).

${ }^{14}$ R. Arenal, A. C. Ferrari, S. Reich, L. Wirtz, J.-Y. Mevellec, S.
Lefrant, A. Rubio, and A. Loiseau, Nano Lett. 6, 1812 (2006).

${ }^{15}$ G. G. Fuentes, E. Borowiak-Palen, T. Pichler, X. Liu, A. Graff, G. Behr, R. J. Kalenczuk, M. Knupfer, and J. Fink, Phys. Rev. B 67, 035429 (2003).

${ }^{16}$ A. Zobelli, C. P. Ewels, A. Gloter, O. Stephan, S. Csillag, and C. Colliex, Nano Lett. 6, 1955 (2006).

${ }^{17}$ D. Golberg, M. Mitome, K. Kurashima, C. Y. Zhi, C. C. Tang, Y. Bando, and O. Lourie, Appl. Phys. Lett. 88, 123101 (2006).

${ }^{18}$ M. Radosavljevic, J. Appenzeller, V. Derycke, R. Martel, P. Avouris, A. Loiseau, J.-L. Cochon, and D. Pigache, Appl. Phys. Lett. 82, 4131 (2003).

${ }^{19}$ R. Czerw, S. Webstern, D. L. Caroll, S. M. C. Vieira, P. R. Birkett, C. A. Rego, and S. Roth, Appl. Phys. Lett. 83, 1617 (2003).

${ }^{20}$ M. Ishigami, J. D. Sau, S. Aloni, M. L. Cohen, and A. Zettl, Phys. Rev. Lett. 94, 056804 (2005).

${ }^{21}$ M. Ishigami, J. D. Sau, S. Aloni, M. L. Cohen, and A. Zettl, Phys. Rev. Lett. 97, 176804 (2006).

${ }^{22}$ C. Zhi, Y. Bando, C. Tang, D. Golberg, Z. Xie, and T. Sekiguchi, Appl. Phys. Lett. 86, 213110 (2005).

${ }^{23}$ J. Wu, W.-Q. Han, W. Walukiewicz, J. W. Ager III, W. Shan, E. E. Haller, and A. Zettl, Nano Lett. 4, 647 (2004).

${ }^{24}$ J. S. Lauret, R. Arenal, F. Ducastelle, A. Loiseau, M. Cau, B. Attal-Trétout, and E. Rosencher, Phys. Rev. Lett. 94, 037405 (2005).

${ }^{25}$ P. Jaffrennou, J. Barjon, J.-S. Lauret, A. Maguer, D. Golberg, B. Attal-Trétout, F. Ducastelle, and A. Loiseau, Phys. Status Solidi 
B 244, 4147 (2007).

${ }^{26}$ P. Jaffrennou, F. Donatini, J. Barjon, J.-S. Lauret, A. Maguer, B. Attal-Trétout, F. Ducastelle, and A. Loiseau, Chem. Phys. Lett. 442, 372 (2007).

${ }^{27}$ W.-Q. Han, H.-G. Yu, C. Zhi, J. Wang, Z. Liu, T. Sekiguchi, and Y. Bando, Nano Lett. 8, 491 (2008).

${ }^{28}$ C. Tang, Y. Bando, T. Sato, and K. Kurashima, Chem. Commun. (Cambridge) 2002, 1290.

${ }^{29}$ C. Y. Zhi, Y. Bando, C. Tang, and D. Golberg, Solid State Commun. 135, 67 (2005).

${ }^{30}$ C. Zhi, Y. Bando, C. Tang, S. Honda, K. Sato, H. Kuwahara, and D. Golberg, J. Phys. Chem. B 110, 1525 (2006).

${ }^{31}$ M. G. Silly, P. Jaffrennou, J. Barjon, J.-S. Lauret, F. Ducastelle, A. Loiseau, E. Obraztsova, B. Attal-Trétout, and E. Rosencher, Phys. Rev. B 75, 085205 (2007).

${ }^{32}$ P. Jaffrennou, J. Barjon, J.-S. Lauret, B. Attal-Trétout, F. Ducastelle, and A. Loiseau, J. Appl. Phys. 102, 116102 (2007).

${ }^{33}$ G. Zimmerer, Nucl. Instrum. Methods Phys. Res. A 308, 178 (1991).

${ }^{34}$ K. Watanabe, T. Taniguchi, and H. Kanda, Nat. Mater. 3, 404 (2004).

${ }^{35}$ B. Berzina, L. Trinkler, V. Korsak, R. Krutohvostov, D. L. Carroll, K. B. Ucer, and R. T. Williams, Phys. Status Solidi B 243, 3840 (2006).

${ }^{36}$ N. Koi, T. Oku, I. Narita, and K. Suganuma, Diamond Relat. Mater. 14, 1190 (2005).

${ }^{37}$ H. Zhang, J. D. FitzGerald, L. T. Chadderton, J. Yu, and Y. Chen, Phys. Rev. B 74, 045407 (2006).

${ }^{38}$ Y.-C. Zhu, Y. Bando, D.-F. Xue, T. Sekiguchi, D. Golberg, F.-F. Xu, and W.-L. Liu, J. Phys. Chem. B 108, 6193 (2004).

${ }^{39} \mathrm{~T}$. Taniguchi and K. Watanabe, J. Cryst. Growth 303, 525 (2007).

${ }^{40}$ J.-S. Lauret, C. Voisin, G. Cassabois, C. Delalande, Ph. Roussignol, O. Jost, and L. Capes, Phys. Rev. Lett. 90, 057404
(2003).

${ }^{41}$ T. J. McDonald, C. Engtrakul, M. Jones, G. Rumbles, and M. J. Heben, J. Phys. Chem. B 110, 25339 (2006).

${ }^{42}$ A. Hartschuh, H. N. Pedrosa, L. Novotny, and T. D. Krauss, Science 301, 1354 (2003).

${ }^{43}$ J. Lefebvre, Y. Homma, and P. Finnie, Phys. Rev. Lett. 90, 217401 (2003).

${ }^{44}$ L. Museur, D. Anglos, J.-P. Petitet, J.-P. Michel, and A. K. Kanaev, J. Lumin. 127, 595 (2007).

${ }^{45}$ K. Watanabe, T. Taniguchi, T. Kuroda, and H. Kanda, Appl. Phys. Lett. 89, 141902 (2006).

${ }^{46}$ K. Watanabe, T. Taniguchi, T. Kurodaa, O. Tsuda, and H. Kanda, Diamond Relat. Mater. 17, 830 (2008).

${ }^{47}$ L. Museur and A. Kanaev, J. Appl. Phys. 103, 103520 (2008).

${ }^{48}$ K. H. Khoo, M. S. C. Mazzoni, and S. G. Louie, Phys. Rev. B 69, 201401(R) (2004).

${ }^{49}$ O. Ambacher, R. Dimitrov, M. Stutzmann, B. E. Foutz, M. J. Murphy, J. A. Smart, J. R. Shealy, N. G. Weimann, K. Chu, M. Chumbes, B. Green, A. J. Sierakowski, W. J. Schaff, and L. F. Eastman, Phys. Status Solidi B 216, 381 (1999).

${ }^{50}$ I. L. Guy, Z. Zheng, M. Wintrebert-Fouquet, K. S. A. Butcher, P. Chen, and T. L. Tansley, J. Cryst. Growth 269, 72 (2004).

${ }^{51}$ J. Simon, N. T. Pelekanos, C. Adelmann, E. Martinez-Guerrero, R. André, B. Daudin, Le Si Dang, and H. Mariette, Phys. Rev. B 68, 035312 (2003).

${ }^{52}$ D. Golberg, Y. Bando, M. Eremets, K. Takemura, K. Kurashima, K. Tamiya, and H. Yusa, Chem. Phys. Lett. 279, 191 (1997).

${ }^{53}$ D. Golberg, M. Mitome, Y. Bando, C. C. Tang, and C. Y. Zhi, Appl. Phys. A: Mater. Sci. Process. 88, 347 (2007).

${ }^{54}$ K. S. Song and R. T. Williams, Self-Trapped Excitons (SpringerVerlag, Berlin, 1993).

${ }^{55}$ Y. Taniyasu, M. Kasu, and T. Makimoto, Nature (London) 441, 325 (2006). 